\title{
The comparison of resting metabolic rate between biopsy-proven non-alcoholic steatohepatitis and non-alcoholic fatty liver patients
}

\author{
(D) Emre Batuhan Kenger ${ }^{1}$, (D) Hakan Guveli ${ }^{1}$, (D) Can Ergun ${ }^{1}$, (D) Eda Kaya ${ }^{2}$, (D) Yusuf Yilmaz
}

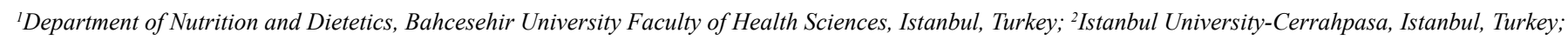
${ }^{3}$ Department of Gastroenterology, Marmara University Faculty of Medicine, Istanbul, Turkey

\begin{abstract}
Background and Aim: Resting metabolic (RMR) rate was shown to be associated with chronic inflammatory conditions. In this study, we aimed to investigate whether RMR differs significantly in patients with non-alcoholic steatohepatitis (NASH) from patients with non-alcoholic fatty liver disease (NAFLD) without evidence of inflammation.

Material and Methods: Forty-two biopsy-proven NASH were compared with 37 NAFLD patients, who had normal serum transaminases and no evidence of fibrosis based on transient elastography examination. In the interviews, patients' levels of physical activity and dietary habits were recorded, and bioimpedance analysis was performed. The RMRs were calculated using an indirect calorimeter.

Results: RMR did not significantly differ between patients with NASH and NAFLD without steatohepatitis in both genders ( $p=0.695$ in males, $p=0.256$ in females). However, only in female patients RMR rate per body weight was significantly higher in patients with NASH $(22.3[17.2-26.6] \mathrm{cal} / \mathrm{kg}$ to $20.2[12.2-26.1] \mathrm{cal} / \mathrm{kg}, \mathrm{p}=0.020)$.

Conclusion: In conclusion, RMR was not significantly associated with steatohepatitis in patients with NAFLD. Considering the minimizing the effects of body weight, RMR rate per body weight may be used over RMR in the evaluation of the inflammatory status of the NAFLD.
\end{abstract}

Keywords: Inflammation; non-alcoholic fatty liver disease; non-alcoholic steatohepatitis; fibroscan; resting metabolic rate.

\section{Introduction}

Non-alcoholic fatty liver (NAFL) disease (NAFLD) is a clinicopathological condition that is characterized by hepatic fat accumulation when other etiologies are excluded. ${ }^{[1]}$ NAFLD may be seen on a wide clinical spectrum ranging from hepatic steatosis to steatohepatitis and even hepatic fibrosis resulted in liver-related morbidity and mortality. ${ }^{[2]}$

Received: December 12, 2019; Accepted: January 07, 2020; Available online: January 17, 2020

Corresponding author: Emre Batuhan Kenger; Bahcesehir Universitesi Saglik Bilimleri Fakultesi, Beslenme ve Diyetetik Bolumu, Ihlamur Yildiz Caddesi, No: 10 Besiktas, Istanbul, Turkey

Phone: +90 21238191 80; e-mail: emrebatuhan.kenger@hes.bau.edu.tr

CC Copyright 2020 by Hepatology Forum - Available online at www.hepatologyforum.org
NAFL is usually the benign histopathological subtype of NAFLD and the development of liver-related morbidity is rarely seen. On the other hand, non-alcoholic steatohepatitis (NASH) leads to inflammation and hepatocellular damage, which has a stronger potential to progress into end-stage liver failure and hepatocellular carcinoma. ${ }^{[1]}$

Although there are ongoing clinical trials, there is no approved pharmacological therapy in NAFLD. The first-line therapeutic option in NAFLD independent from the histopathological form still remains as loss of weight and prevention of weight gain, as well as lifestyle changes, including a healthy diet and regular physical activity. ${ }^{[3]}$ Many clinical studies showed that slight and moderate loss of weight improved insulin sensitivity, liver transaminase levels and hepatic steatosis. ${ }^{[4-6]}$ At least a $7-10 \%$ loss in body weight could even lead to the resolution of NASH and regression in the fibrosis stage. ${ }^{[7]}$

In the prescription of a diet, the energy requirement constructs the cornerstone of the nutritional recommendation. The energy requirement is defined as the amount of nutrients that an individual should take daily based on age, sex, body weight, height, and the level of physical activity to grow or to survive. Body weight is an indication of whether the energy intake is sufficient. ${ }^{[8]}$ Energy is consumed by the human body as defined by the basal metabolism rate (BMR), thermic effects of food, and activity thermogenesis. These three components constitute the total energy expenditure. The resting metabolic rate (RMR) is the amount of energy that the body needs to maintain homeostasis. RMR does not include thermogenesis, physical activity, or other components of energy expenditure, and is approximately $10-20 \%$ higher than BMR. ${ }^{[9]}$ It is stated that a low and/or high level of RMR may be associated with various comorbidities. ${ }^{[10]}$ Low levels of RMR can be a risk factor for metabolic syndrome ${ }^{[11]}$ and insulin resistance, ${ }^{[12]}$ whereas low RMR has a negative impact on the metabolic profile in obese individuals. ${ }^{[10]}$ For the comparison of patients with various body sizes to adjust the effects of increased weight on RMR or RMR per $\mathrm{kg}$, body weight was also demonstrated for further use. ${ }^{[10]}$

NAFLD, which triggers metabolic changes in metabolism, is closely associated with obesity, metabolic syndrome, and insulin resistance. ${ }^{[13]}$ However, to our knowledge, there is no formal study that shows the relationship between the histopathological status of NAFLD and RMR. In this study, we aimed to investigate whether RMR is significantly different between NASH and NAFLD without evidence of steatohepatitis patients. 


\section{Materials and Methods}

\section{Patients}

A total of 79 patients, who were presented to their routine follow-ups in Marmara University Institute of Gastroenterology between December 2017 and March 2018, were enrolled in this study. The patients who were followed up under the diagnosis of NAFLD with a body mass index $(B M I) \geq 25 \mathrm{~kg} / \mathrm{m}^{2}$ and volunteered to participate in this study were included. The exclusion criteria were as follows: having smoked in the past one hour, having performed heavy physical activity up to 24 hours ago, having eaten food up to four hours ago, having alcoholic liver disease, having chronic obstructive pulmonary disease, having drunk tea or coffee up to four hours ago, and being in the menstruation period for female patients. The participants' demographic data, clinical and biochemical findings were obtained from the patients' files. A BMI of $\geq 25 \mathrm{~kg} / \mathrm{m}^{2}$ was defined as overweight.

The study patients consisted of two groups as follows: 1) biopsy-proven NASH patients 2) NAFLD patients, in whom hepatic steatosis was concluded according to transient elastography (TE) examination and diagnosis of NASH was excluded in the absence of elevated liver transaminases and absence of fibrosis in TE examinations.

\section{Data Collection}

The data regarding nutritional habits were collected with face-to-face interviews. The dietary status of the individuals was determined using the food consumption recording method while their physical activity status was determined according to the International Physical Activity Questionnaire. ${ }^{[14]}$ Body composition data were obtained using bioimpedance analysis via Inbody $120 \mathrm{R}$ according to the manufacturer's instructions. Waist and hip circumferences were measured using a non-stretching measuring tape.

\section{Indirect Calorimeter Measurement}

In the indirect calorimeter measurement method, the individual's oxygen consumption and carbon dioxide production are measured for a certain period of time. The Weir equation and the fixed respiratory coefficient value of 0.85 are used to convert oxygen consumption to RMR. ${ }^{[15]}$ Attention was paid to ensure that the individual whose values were measured had been hungry for four-five hours, had not smoked or drunk alcohol up to two hours before this measurement, had not done moderate exercise up to two hours previous, and had not performed heavy exercise up to 14 hours before this measurement. The measurements took for 15 minutes with the individual in a resting position. ${ }^{[16]}$

\section{Fibroscan Examinations}

All the Fibroscan examinations were performed using Fibroscan 502 touch device following the manufacturer's instructions (Echosens SA, Paris, France) by a single operator (YY). The examinations were started with $\mathrm{M}$ probe. The probe was switched to XL following the automatic probe selection tool displayed in real-time, which is based on the skin to liver capsule distance. The patients were placed in the dorsal decubitus position, and the transducer probe was positioned in the intercostal space of the right lobe of the liver. The reliable TE measurement was defined as at least 10 valid measurements and having an interquartilerange-to-median ratio of $\leq 0.3 .{ }^{[17]}$ Controlled attenuation parameter (CAP) was used for estimation of hepatic steatosis and liver stiffness measurement (LSM) for liver fibrosis. The information about the mea- surement of LSM and CAP in TE was as provided in detail previously. A CAP cutoff of $>238 \mathrm{~dB} / \mathrm{m}$ indicated hepatic steatosis. ${ }^{[18]}$ An LSM $>7$ $\mathrm{kPa}$ was used for the exclusion of the presence of fibrosis. ${ }^{[19]}$

\section{Liver Histology}

The liver biopsy conditions were described in detail previously. ${ }^{[20]}$ The liver biopsy specimens were evaluated according to two approved scores: The specimens were scored according to the Steatosis, Activity and Fibrosis/Fatty Liver Inhibition of Progression histological algorithm and categorized into non-NASH and $\mathrm{NASH}^{[21]}$ by a pathologist expertized in the liver.

\section{Statistical Analysis}

The statistical analysis conducted as male and female subjects separately. The categorical data were presented as counts and percentages and continuous data as median [minimum-maximum]. The categorical variables were assessed using the chi-square test. Due to the small number of the groups, continuous variables were assessed using the nonparametric tests. Continuous variables were compared using the Man$\mathrm{n}$-Whitney $\mathrm{U}$ test. The statistical analysis was conducted using SPSS 22.0 , and $\mathrm{p}<0.05$ was considered statistically significant.

\section{Ethics}

This study was approved by the local ethics committee (Bahcesehir University Clinical Research Ethics Committee. Approval date: 4.10.2017, Approval number: 2017-15/03) and in adherence to the Declaration of Helsinki. Financial support was provided by Marmara University Institute of Gastroenterology. Informed consent was obtained from all the patients.

\section{Results}

This study consisted of 79 patients (41 male, 38 female). The general characteristics of the study patients are depicted in Table 1 separately analyzed according to gender. Of the 41 male patients, $30(73.2 \%)$ of them had NASH, and $11(26.8 \%)$ of them had NAFLD, whereas from 38 female patients $12(31.6 \%)$ of them had NASH and $26(61.8 \%)$ of them had NAFLD. In both gender groups, the consumption amount of macronutrients usually did not significantly differ between patients with NASH and NAFLD, as shown in Table 2. Only female NAFLD patients consumed significantly more amount of fat than female patients with NASH $(\mathrm{p}=0.008)$. Among both genders, both patients with NASH and NAFLD had mostly a sedentary lifestyle. Among males, $73.3 \%(n=22)$ of the NASH patients and $81.8 \%(n=9)$ of the NAFLD patients $(\mathrm{p}=0.700)$ and among females, $75 \%(\mathrm{n}=9)$ of the NASH patients and $92.3 \%(n=24)(\mathrm{p}=0.301)$ had low physical activity level.

The histological characteristics of the NASH patients are presented in Table 3. Significant fibrosis was present in $56.7 \%(n=17)$ among male patients and $83.3 \%(\mathrm{n}=10)$ female patients $(\mathrm{p}=0.147)$ and advanced fibrosis in $16.7 \%(\mathrm{n}=5)$ and in $58.3 \%(\mathrm{n}=7)$, respectively $(\mathrm{P}=0.015)$. Among males, the median LSM was measured as 9.7 [5.5-24.0] $\mathrm{kPa}$ and 4.8 [4.2$6.5] \mathrm{kPa}(\mathrm{p}<0.001)$ among NASH and NAFLD patients, respectively and CAP as 329 [113-400] dB/m and 313 [242-400] dB/m ( $\mathrm{p}=0.441)$, respectively. Among females, median LSM was 11.3 [6.0-14.6] $\mathrm{kPa}$ and $4.5[3.1-6.0] \mathrm{kPa}(\mathrm{p}<0.001)$ for NASH and NAFLD patients, respectively and CAP 320 [272-361] dB/m and 305 [248-400] dB/m ( $\mathrm{p}=0.174)$, respectively. The TE measurements are visualised in Figure 1. 


\begin{tabular}{|c|c|c|c|}
\hline \multicolumn{4}{|c|}{ Age (years) } \\
\hline 47 [26-64] & 52 [26-63] & 47 [26-64] & 0.360 \\
\hline 57 [36-65] & 49 [34-75] & 53 [34-75] & 0.053 \\
\hline \multicolumn{4}{|c|}{ Type 2 diabetes mellitus (yes/no) } \\
\hline $18 / 12$ & $1 / 10$ & $19 / 22$ & 0.004 \\
\hline $12 / 18$ & $1 / 10$ & $13 / 28$ & 0.060 \\
\hline $8 / 4$ & $4 / 22$ & $12 / 26$ & 0.002 \\
\hline \multicolumn{4}{|c|}{ Dyslipidemia (yes/no) } \\
\hline $28 / 2$ & $10 / 1$ & $38 / 3$ & 0.792 \\
\hline $10 / 2$ & $18 / 8$ & $28 / 10$ & 0.359 \\
\hline \multicolumn{4}{|c|}{$\mathrm{BMI}\left(\mathrm{kg} / \mathrm{m}^{2}\right)$} \\
\hline $104[93-119]$ & $103[85-123]$ & $104[85-123]$ & 0.718 \\
\hline \multicolumn{4}{|c|}{ Hip circumference (cm) } \\
\hline $110[98-122]$ & $110[102-136]$ & $110[98-136]$ & 0.565 \\
\hline $106[96-116]$ & $114[92-144]$ & $111[92-144]$ & 0.014 \\
\hline \multicolumn{4}{|c|}{ AST (U/L) } \\
\hline $46[13-103]$ & $22[16-29]$ & 34 [13-103] & $<0.001$ \\
\hline $46[18-130]$ & $22[13-33]$ & $23[13-130]$ & $<0.001$ \\
\hline \multicolumn{4}{|c|}{ ALT (U/L) } \\
\hline 92 [19-209] & 28 [20-36] & 54 [19-209] & $<0.001$ \\
\hline $50[24-217]$ & $19[12-38]$ & $25[12-217]$ & $<0.001$ \\
\hline \multicolumn{4}{|c|}{ Albumin (mg/dL) } \\
\hline $4.7[3.5-5.9]$ & $4.6[4.4-4.8]$ & $4.7[3.5-5.9]$ & 0.198 \\
\hline $45[28-65]$ & $44[30-55]$ & 45 [28-65] & 0.453 \\
\hline $48[36-63]$ & 48 [29-65] & $48[29-65]$ & 0.588 \\
\hline \multicolumn{4}{|c|}{$\mathrm{LDL}(\mathrm{mg} / \mathrm{dL})$} \\
\hline 119 [72-266] & $130[108-212]$ & 122 [72-266] & 0.223 \\
\hline $104[45-152]$ & $145[73-202]$ & 131 [45-202] & 0.976 \\
\hline \multicolumn{4}{|c|}{ Glucose (mg/dL) } \\
\hline 98 [80-163] & 99 [86-121] & 98 [80-163] & 0.802 \\
\hline $112[86-189]$ & 96 [77-185] & $102[77-189]$ & 0.013 \\
\hline \multicolumn{4}{|c|}{ Body fat mass (kg) } \\
\hline $28.9[17.4-46.1]$ & $24.2[21.2-55.3]$ & $27.9[17.4-55.3]$ & 0.837 \\
\hline $31.5[19.9-42.3]$ & $32.7[20.8-64.2]$ & $32.4[19.9-64.2]$ & 0.510 \\
\hline \multicolumn{4}{|c|}{ Body fat ratio \% } \\
\hline $31.2[21.5-39.0]$ & 29.4 [24.6-42.2] & 29.6 [21.5-42.2] & 0.648 \\
\hline $40.7[29.7-53.1]$ & $41.6[31.3-53.5]$ & $40.7[29.7-53.5]$ & 0.272 \\
\hline \multicolumn{4}{|c|}{ Body muscle mass $(\mathrm{kg})$} \\
\hline $36.5[31.2-44.8]$ & $40.3[33.9-61.8]$ & $37.3[31.2-61.8]$ & 0.041 \\
\hline $26.2[17.4-53.6]$ & 26.0 [21.5-55.9] & 36 [17.4-55.9] & 0.561 \\
\hline & & & \\
\hline $40.0[34.5-45.2]$ & 41.0 [33.0-71.9] & $40.3[33.0-71.9]$ & 0.462 \\
\hline $33.2[26.3-60.2]$ & $33.7[27.1-58.0]$ & $33.4[26.4-60.2]$ & 0.572 \\
\hline
\end{tabular}

NASH: Non-alcoholic steatohepatitis; NAFL: Non-alcoholic fatty liver; BMI: Body mass index; AST: Aspartate transaminase; ALT: Alanine transaminase; HDL: High density

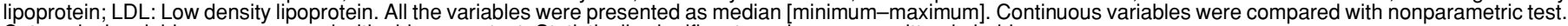
Categorical variables are compared with chi-square test. Statistically significant p-values were written in bold. 


\begin{tabular}{|c|c|c|c|c|}
\hline \multicolumn{5}{|l|}{ Energy (kcal) } \\
\hline Female & 1650 [1506-1958] & 1698 [1222-2570] & 1667 [1222-2570] & 0.683 \\
\hline \multicolumn{5}{|l|}{ Protein (gr) } \\
\hline Male & 77.4 [54.8-113.2] & $92.5[56.3-114.4]$ & $82.5[54.8-114.4]$ & 0.083 \\
\hline Male & $78.9[60.2-127.1]$ & $82.1[65.2-118.1]$ & $85.6[60.2-127.1]$ & 0.780 \\
\hline Female & $71.1[55.1-92.3]$ & $87.3[49.0-128.5]$ & $75.9[49.0-128.5]$ & 0.008 \\
\hline \multicolumn{5}{|c|}{ Carbohydrate (gr) } \\
\hline Male & $193.3[118.6-308.7]$ & $203.3[141.6-317.9]$ & 195.1 [118.6-317.9] & 0.257 \\
\hline Female & 184.4 [122.2-222.2] & 159.4 [119.8-315.3] & 168.6 [119.8-315.3] & 0.064 \\
\hline \multicolumn{5}{|l|}{ Protein (\%) } \\
\hline Female & $37.0[31.0-53.0]$ & $44.5[33.0-54.0]$ & $40.5[31.0-54.0]$ & 0.009 \\
\hline \multicolumn{5}{|c|}{ Carbohydrate (\%) } \\
\hline Male & 40.0 [32.0-52.0] & 44.0 [33.0-50.0] & $42.0[32.0-52.0]$ & 0.479 \\
\hline Female & $45.0[32.0-52.0]$ & $39.5[30.0-53.0]$ & $42.5[30.0-53.0]$ & 0.029 \\
\hline
\end{tabular}

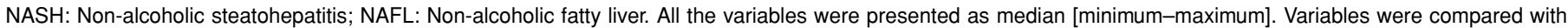
nonparametric test. Statistically significant $p$-values were written in bold.

Table 3. Histological characteristics of the non-alcoholic steatohepatitis patients

\begin{tabular}{llll}
\hline Variables & Male $(\mathbf{n}=\mathbf{3 0})$ & Female $(\mathbf{n}=\mathbf{1 2})$ & $\mathbf{p}$ \\
\hline Steatosis grade S1/S2/S3, \% & $4(13.3) / 8(26.7) / 18(60)$ & $0(0) / 4(33.3) / 8(66.7)$ & 0.408 \\
Activity grade A1/A2/A3/A4, \% & $1(3.3) / 11(36.7) / 10(33.3) / 8(26.7)$ & $0(0) / 1(8.3) / 10(83.3) / 1(8.3)$ \\
Fibrosis stage F1/F2/F3/F4, \% & $1(3.3) / 12(40) / 12(40) / 4(13.3) / 1(3.3)$ & $0(0) / 2(16.7) / 3(25) / 6(50) / 1(8.3)$ & 0.034 \\
\hline Categorical variables are compared with Chi Square test. Statistically significant p-values were written in bold. & 0.106 \\
\hline
\end{tabular}

\begin{tabular}{|c|c|c|c|c|}
\hline & Controlled attenuation parameter $(\mathrm{dB} / \mathrm{m})$ & Liver stiffness measurement $(\mathrm{kPa})$ \\
330 \\
325 \\
320 \\
315 \\
310 \\
305 \\
300 \\
295
\end{tabular}

Figure 1. Transient elastography measurement results.

When RMR values between NASH and NAFLD patients compared, among male individuals, the median RMR was 1987 [1347-2522] cal and 2044 [1548-2466] cal in NASH and NAFLD patients, respectively. Among female individuals, the median RMR was found as 1644 [13032011] cal in NASH patients and 1542 [1174-2420] cal in NAFLD patients. Both among male $(p=0.695)$ and female $(p=0.256)$ NASH and
NAFLD patients, no significant difference was detected. Among males, RMR per kg did not show any significant difference between patients with NASH (21.1 [15.0-28.3] cal/kg) and NAFLD (21.1 [15.6-24.4] $\mathrm{cal} / \mathrm{kg}),(\mathrm{p}=0.746)$. However, among females, the difference in RMR per $\mathrm{kg}$ between patients with NASH (22.3 [17.2-26.6] cal $/ \mathrm{kg})$ and NAFLD $(20.2[12.2-26.1] \mathrm{cal} / \mathrm{kg})$ was statistically significant $(\mathrm{p}=0.020)$. 


\section{Discussion}

In this study, we compared for the first time RMR of the patients with NASH and NAFLD without evidence of steatohepatitis and found that there was no significant association between having NASH and RMR value among patients with the same gender. However, only among female patients, we found a statistically significant difference between RMR per body weight values in comparison to patients with NASH and NAFLD.

A Westernized diet consisted of high-calorie intake, mostly saturated fats, refined carbohydrates and fructose is associated with increased risk of weight gain, obesity and NAFLD..$^{[22]}$ Moreover, an unhealthy diet, including high-calorie intake, obesity and a sedentary lifestyle, are more commonly observed among individuals with NAFLD ${ }^{[23]}$ In this context, NAFLD was shown to be strongly associated with the presence of metabolic syndrome, type 2 diabetes mellitus and hypertension and the likelihood of NASH increases parallelly to the number of accompanying comorbidities. ${ }^{[24]}$ As reported previously, both obesity and type 2 diabetes mellitus are characterized by impaired insulin sensitivity and low-grade inflammation. ${ }^{[25]}$ Furthermore, in obese patients with impaired glucose tolerance was associated with higher levels of RMR compared to obese patients without impaired glucose metabolism. ${ }^{[26,27]}$ Considering the significantly positive association between RMR and C-reactive protein level, which is an acute-phase protein, was reported among patients with chronic diseases, ${ }^{[28,29]}$ which may be further explained by increased energy costs in the presence of inflammation as in NASH. ${ }^{[30]}$ However, in our study, we could not provide an implicit evidence for a positive association with RMR and NASH. This could be further explained by the effects of age and accompanying chronic diseases on the RMR status of the patients, although we evaluated the RMR status gender-specific to minimize this interfering effect. On the other hand, following the positive association between obesity and RMR, ${ }^{[26,27]}$ the concept of the RMR per body weight may be more useful in the evaluation of energy consumption status and indirectly inflammatory status of the NAFLD patients by minimizing the effects of body weight.

There are some limitations to this study. Firstly, the number of patients recruited in the present study was relatively low. Secondly, due to the ethical reasons, we could not confirm the diagnosis of NAFL in patients with NAFLD without evidence of steatohepatitis with liver biopsy in those patients who were recruited for this study with the absence of NASH. Thirdly, we did not match patients according to comorbid diseases, such as hypertension and type 2 diabetes mellitus, which may significantly affect the comparison of the patients' metabolic rate. Despite all of these limitations, this work contributes to the literature providing an assessment of RMR between patients with NAFLD and NASH both in males and females.

\section{Conclusion}

In conclusion, RMR was not significantly associated with NASH, which indicates inflammation of liver among NAFLD patients. Only RMR per body weight in females remained statistically higher in NASH patients compared to NAFLD without evidence of steatohepatitis. Our findings suggest that RMR may be neglected in the prescription of diet to NAFLD patients. However, considering the relationship between increased RMR in other chronic diseases, which is in close relationship with NASH, ${ }^{[10-12]}$ further studies with a larger population should be designed for more accurate results.
Ethics Committee Approval: This study was approved by the local ethics committee (Bahcesehir University Clinical Research Ethics Committee. Approval date: 4.10.2017, approval number: 2017-15/03).

Peer-review: Externally peer-reviewed.

Author Contributions: Concept - EBK, HG; Design - EBK, EK, HG; Supervision - YY; Data Collection and/or Processing - EBK, EK; Analysis and/or Interpretation - EBK, EK, CE; Literature Search - EBK, EK; Writing - EBK, EK, CE; Critical Reviews - YY.

Conflict of Interest: The authors have no conflict of interest to declare.

Financial Disclosure: This study was supported by Marmara University Institute of Gastroenterology.

\section{References}

1. Yilmaz Y, Younossi ZM. Obesity-associated nonalcoholic fatty liver disease. Clin Liver Dis 2014;18(1):19-31.

2. Byrne CD, Targher . NAFLD: a multisystem disease. J Hepatol 2015;62 (Suppl 1):S47-64.

3. Zelber-Sagi S, Godos J, Salomone F. Lifestyle changes for the treatment of nonalcoholic fatty liver disease: a review of observational studies and intervention trials. Therap Adv Gastroenterol 2016;9(3):392-407.

4. Colak Y, Tuncer I, Senates E, Ozturk O, Doganay L, Yilmaz Y. Nonalcoholic fatty liver disease: a nutritional approach. Metab Syndr Relat Disord 2012;10(3):161-166.

5. Kim NH, Kim JH, Kim YJ, Yoo HJ, Kim HY, Seo JA, et al. Clinical and metabolic factors associated with development and regression of nonalcoholic fatty liver disease in nonobese subjects. Liver Int 2014;34(4):604-611.

6. Wong VW, Wong GL, Chan RS, Shu SS, Cheung BH, Li LS, et al. Beneficial effects of lifestyle intervention in non-obese patients with non-alcoholic fatty liver disease. J Hepatol 2018;69(6):1349-1356.

7. Brunner KT, Henneberg CJ, Wilechansky RM, Long MT. Nonalcoholic Fatty Liver Disease and Obesity Treatment. Curr Obes Rep 2019;8(3):220228.

8. Kee AL, Isenring E, Hickman I, Vivanti A. Resting energy expenditure of morbidly obese patients using indirect calorimetry: a systematic review. Obes Rev 2012;13(9):753-765.

9. Ireton-Jones CS. Intake: Energy. In: Mahan LK, Raymond JL, editors. Krause's Food \& The Nutrition Care Process. St. Louis, Missouri: Elsevier; 2017. p. 17-27.

10. Hosseini B, Mirzaei K, Maghbooli Z, Keshavarz SA, Hossein-Nezad A. Compare the resting metabolic rate status in the healthy metabolically obese with the unhealthy metabolically obese participants. J Nutr Intermed Metab 2016;6:48-53.

11. Buscemi S, Verga S, Caimi G, Cerasola G. A low resting metabolic rate is associated with metabolic syndrome. Clin Nutr 2007;26(6):806-809.

12. Georgopoulos NA, Saltamavros AD, Vervita V, Karkoulias K, Adonakis G, Decavalas G, et al. Basal metabolic rate is decreased in women with polycystic ovary syndrome and biochemical hyperandrogenemia and is associated with insulin resistance. Fertil Steril 2009;92(1):250-255.

13. Oliveira CP, de Lima Sanches P, de Abreu-Silva EO, Marcadenti A. Nutrition and physical activity in nonalcoholic fatty liver disease. J Diabetes Res 2016;2016:4597246.

14. Hagströmer M, Oja P, Sjöström M. The International Physical Activity Questionnaire (IPAQ): a study of concurrent and construct validity. Public Health Nutr 2006;9(6):755-762.

15. Nieman DC, Austin MD, Benezra L, Pearce S, McInnis T, Unick J, et al. Validation of Cosmed' s FitMate in measuring oxygen consumption and estimating resting metabolic rate. Res Sports Med 2006;14(2):89-96.

16. Compher C, Frankenfield D, Keim N, Roth-Yousey L; Evidence Analysis Working Group. Best practice methods to apply to measurement of resting metabolic rate in adults: a systematic review. J Am Diet Assoc 
2006;106(6):881-903

17. Boursier J, Zarski JP, de Ledinghen V, Rousselet MC, Sturm N, Lebail B, et al. Determination of reliability criteria for liver stiffness evaluation by transient elastography. Hepatology 2013;57(3):1182-1191.

18. Kaya E, Demir D, Alahdab YO, Yilmaz Y. Prevalence of hepatic steatosis in apparently healthy medical students: a transient elastography study on the basis of a controlled attenuation parameter. Eur J Gastroenterol Hepatol 2016;28(11):1264-167.

19. Castera L, Forns X, Alberti A. Non-invasive evaluation of liver fibrosis using transient elastography. J Hepatol 2008 May;48(5):835-847.

20. Yılmaz Y, Kanı HT, Demirtaş CÖ, Kaya E, Sapmaz AF, Qutranji L, et al. Growing burden of nonalcoholic fatty liver disease in Turkey: A single-center experience. Turk J Gastroenterol 2019;30(10):892-898.

21. Bedossa P, Poitou C, Veyrie N, Bouillot JL, Basdevant A, Paradis V, et al. Histopathological algorithm and scoring system for evaluation of liver lesions in morbidly obese patients. Hepatology 2012;56(5):1751-1759.

22. Barrera F, George J. The role of diet and nutritional intervention for the management of patients with NAFLD. Clin Liver Dis 2014;18(1):91-112.

23. Gerber L, Otgonsuren M, Mishra A, Escheik C, Birerdinc A, Stepanova $\mathrm{M}$, et al. Non-alcoholic fatty liver disease (NAFLD) is associated with low level of physical activity: a population-based study. Aliment Pharmacol
Ther 2012;36(8):772-781.

24. Younossi Z, Anstee QM, Marietti M, Hardy T, Henry L, Eslam M, et al. Global burden of NAFLD and NASH: trends, predictions, risk factors and prevention. Nat Rev Gastroenterol Hepatol 2018;15(1):11-20.

25. Wellen KE, Hotamisligil GS. Inflammation, stress, and diabetes. J Clin Invest 2005;115(5):1111-1119.

26. Alawad AO, Merghani TH, Ballal MA. Resting metabolic rate in obese diabetic and obese non-diabetic subjects and its relation to glycaemic control. BMC Res Notes 2013;6:382.

27. Weyer C, Bogardus C, Pratley RE. Metabolic factors contributing to increased resting metabolic rate and decreased insulin-induced thermogenesis during the development of type 2 diabetes. Diabetes 1999;48:1607-1614.

28. Utaka S, Avesani CM, Draibe SA, Kamimura MA, Andreoni S, Cuppari L. Inflammation is associated with increased energy expenditure in patients with chronic kidney disease. Am J Clin Nutr 2005;82(4):801-805.

29. Hickmann CE, Roeseler J, Castanares-Zapatero D, Herrera EI, Mongodin A, Laterre PF. Energy expenditure in the critically ill performing early physical therapy. Intensive Care Med 2014;40(4):548-555.

30. Keane KN, Calton EK, Cruzat VF, Soares MJ, Newsholme P. The impact of cryopreservation on human peripheral blood leucocyte bioenergetics. Clin Sci (Lond) 2015;128(10):723-733. 